\title{
A Case of Mistaken Identity: Spinal Epidural Angiolipoma
}

\author{
Faizal A. Haji, Yatri K. Patel, Lee C. Ang, Joseph F. Megyesi
}

Can J Neurol Sci. 2011; 38: 357-359

Angiolipomas are benign neoplasms that appear as painful subcutaneous nodules in the forearm, trunk, or neck. Histopathologically, they are postulated to represent an intermediate entity in the spectrum between lipoma and hemangioma. ${ }^{1}$ Angiolipomas rarely occur in the spinal cord and generally produce a slowly progressive myelopathy or radiculopathy from extrinsic spinal cord or nerve root compression. $^{2-4}$ We present the clinical, radiological, and histological characteristics of a patient with progressive myelopathy who subsequently underwent surgical resection of a dorsally located thoracic spinal epidural angiolipoma.

\section{Case Presentation}

A 65-year-old overweight right-handed female with an unremarkable past medical history presented to our hospital with a nine-month history of bilateral, symmetrical, progressive numbness and burning in her feet, progressing cranially to the level of the umbilicus by the time of admission. Four months after the onset of her symptoms, the patient began having difficulties ambulating, with stiffness and weakness bilaterally in the lower extremities causing multiple falls. During hospitalization, she developed urinary hesitancy with no frank incontinence. Initial physical examination confirmed thoracic myelopathy, with mild symmetric paraparesis, hyperreflexia and upgoing plantar responses in the lower extremity. A sensory level for pinprick and vibration sense was noted at the T8 level.

Computed tomography (CT) of the lumbar spine showed mild degenerative disc disease and an incidental left renal mass suspicious for malignancy. Subsequent magnetic resonance imaging (MRI) with midline cuts through the cervical and thoracic spine demonstrated an iso to hyperintense lesion compared to adjacent soft tissue at T5-7, causing significant compression of the spinal cord and a smaller T4/5 lesion with extension into the left neuroforamen (Figure 1). Both lesions were contrast enhancing. Based on the radiographic features and known prior left renal mass, the pre-operative diagnosis was metastasis versus lymphoma, with a low likelihood of a meningioma or peripheral nerve sheath tumor.

The patient underwent posterior thoracic laminectomy T3-T7 for spinal cord decompression and resection of the epidural masses. Intraoperatively, the surgical site was hypervascular. Following the laminectomy, a large volume of soft, highly vascular, epidural fat was encountered, with the lesion embedded within. No tumor capsule could be identified. Scalloping of bone edges at T6 and increased vascularity of the adjacent bone was also noted, with some evidence of invasion of the lesion into the adjacent lamina. The lesion was completely resected en bloc by
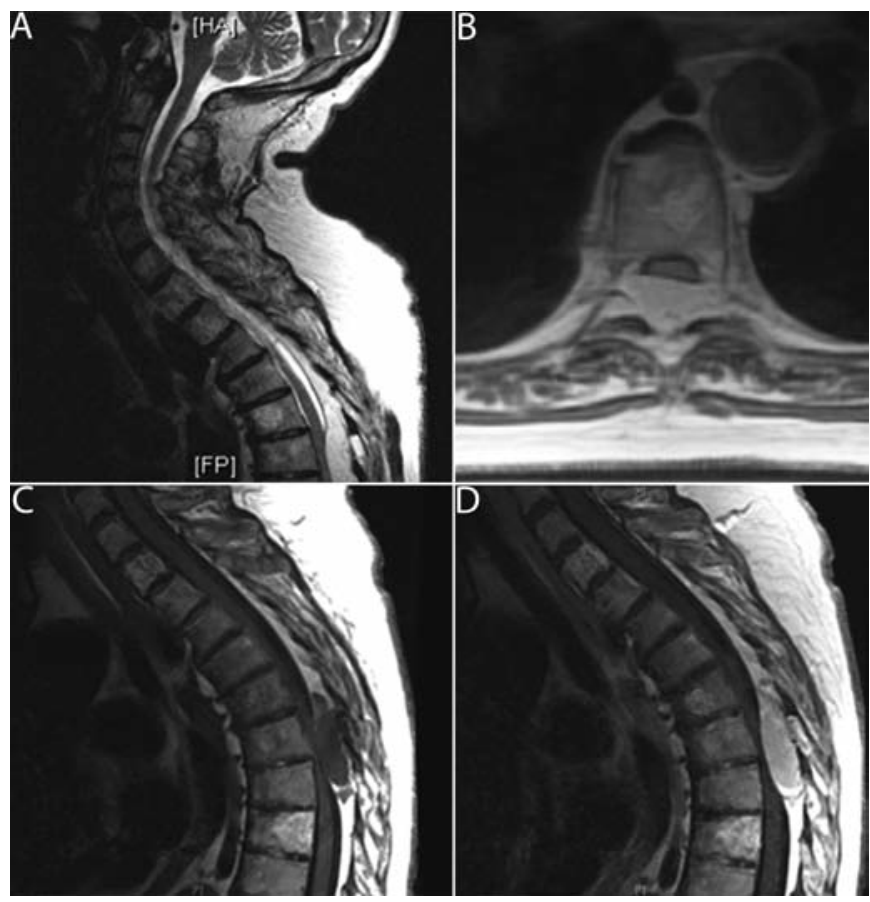

Figure 1: MRI of the patient. (A) T2 weighted sagittal MRI with midline cuts through the cervico-thoracic spine, demonstrating a lesion that is iso to hyperintense compared with adjacent soft tissues at T5-7, causing significant compression of the spinal cord. (B) axial cut through the level of interest demonstrating the level of cord compression. $(C)$ the lesion on $T 1$ pre-contrast images, demonstrating heterogeneous hypointensity of the lesion relative to epidural fat. (D) post contrast, the lesion avidly enhances.

blunt dissection from the underlying dura and sent for frozen and permanent section, with removal of the affected bony elements as well. Pathology demonstrated a lesion consisting of vessels of variable size, including large and dilated thin-walled vasculature lined by "bland" looking endothelial cells. Intermingling between the vascular channels were clusters of matured

From the Department of Clinical Neurological Sciences (FAH, LCA, JFM); Department of Pathology, Division of Neuropathology (LCA); Division of

Neurosurgery (FAH, JFM); Schulich School of Medicine and Dentistry (FAH, YKP); University of Western Ontario, London, Ontario, Canada.

Received April 30, 2010. Final Revisions Submitted September 16, 2010. Correspondence to: Joseph Megyesi, London Health Sciences Centre, University Campus, 339 Windermere Road, London, Ontario, N6A 5A5, Canada. 


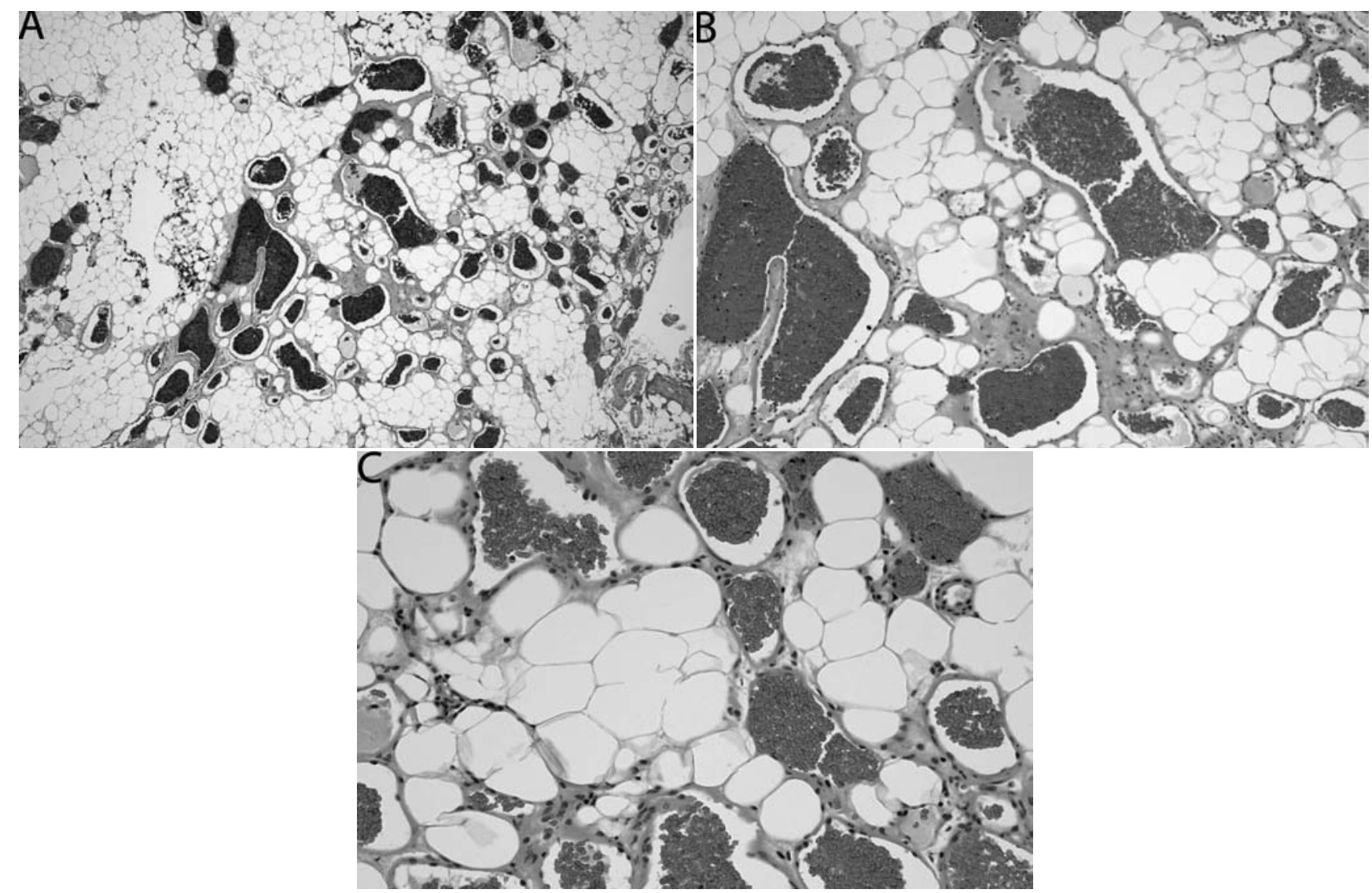

Figure 2: $H \& E 40 X(A), 100 X(B)$ and $200 X(C)$ demonstrating mature, normal appearing adipose tissue intermixed with vascular channels of varying size and shape, with an almost "angioid" appearance with the vessels appearing in clusters. The endothelium of the vessels appears bland and some fibrous connective tissue is demonstrated between the adipose and vascular elements of the lesion. These characteristics are most consistent with an angiolipoma.

adipocytes with some fibrous connective tissue (Figure 2). Overall, the histopathology was consistent with benign angiolipoma, requiring no further adjuvant therapy. Postoperatively, the patient's weakness resolved however she had persistent sensory symptoms below T8. Her ambulation improved and she was able to walk with a cane at the time of discharge. Post-op MRI demonstrated total resection of lesion with persistent increased cord signal at T6 consistent with myelomalacia. In follow-up, her renal mass was found to be a papillary urothelial carcinoma, and she was referred to the Urology service for further management of this incidentally found lesion. She has been followed as an outpatient and yearly MRI imaging is planned.

\section{DISCUSSION}

Angiolipomas are uncommon benign neoplasms rarely occurring in the spinal canal. Spinal angiolipomas (SALs) comprise $0.04-1.2 \%$ of all spinal neoplasms, primarily occurring in the mid-thoracic region. These lesions demonstrate a predilection for females between the fourth and sixth decades of life. ${ }^{2-4}$

The first documented case of SAL, reported by Berenbruch in 1890, was in a 16-year-old male with multiple cutaneous angiolipomas. ${ }^{5}$ The first case of primary SAL was described a decade later by Liebscher, ${ }^{6}$ however the term angiolipoma was not established until 1960 when Howard and Helwig described the anatomopathological characterstics of the lesion containing mature adipocytes admixed with abnormal, proliferative vasculature. ${ }^{7}$ A recent review found 123 reported cases of SAL to date, $95 \%$ occurring in the epidural space and $5 \%$ occurring intramedullary. 8 Early authors postulate that their thoracic tendency may be related to the relative paucity of arterial perfusion in this area, as neovascularization in ischaemic tissues is thought to contribute to the formation of the lesion. ${ }^{7}$

Although their etiology is unclear, SALs are believed to arise from pluripotential mesenchymal stem cells following an undefined stimulus, subsequently differentiating along both angioid and adipose cell lines. ${ }^{10}$ Thus they may represent an intermediate entity between lipomas and hemangiomas. Alternatively, they may represent congenital malformations or benign hamartomas that enlarge with body tissue growth or in response to injury or inflammation. ${ }^{11}$

Two types of primary of SAL have been described: infiltrating and non-infiltrating. Infiltrating lesions are most commonly ventrally located in relation to the cord, demonstrating a locally aggressive nature with invasion into 
adjacent bony, muscular, neural and fibrocollagenous tissues. ${ }^{4}$ Dorsally located lesions are typically non-infiltrating, encapsulated tumors although increased vascularity and scalloping of the adjacent pedicle and lamina is often demonstrated intraoperatively. Interestingly in our case, elements of bony invasion were identified at the time of surgery, which to our knowledge has not been reported in dorsally located lesions previously.

The clinical presentation of SAL is initially insidious in keeping with a slow growing mass. Compression of the spinal cord, cauda equina, or nerve roots, is dependent on the anatomic location of the lesion. ${ }^{1,10}$ Spinal angiolipomas most commonly presents with progressive or sudden weakness and altered sensation in the lower extremities $(40 \%)$, associated with thoracic or lumbar pain (20\%), radicular symptoms, and gait and sphincter disturbance. They may have a relapsing-remitting course or a sudden deterioration, presumably relating to vascular engorgement, hemorrhage into the tumor, or vascular steal. ${ }^{12}$ As in our patient, obesity is believed to be a significant risk factor. Pregnancy is an additional risk factor, possibly relating to hormonal influences on adipocity and tumor growth. ${ }^{13}$

The SAL imaging pattern varies depending on the ratio between vascular and adipose elements of the lesion. Plain XRay may be normal or in infiltrative cases may show vertical trabeculation of adjacent bone similar to the pattern seen in hemangiomas. Erosion of the pedicle and widening of spinal canal has also been described. On CT scan, the lesion appears hypo- or hyper-dense compared to adjacent tissue depending on the degree of adipocity or vascularity. Magnetic resonance imaging is most helpful in distinguishing SAL from other lesions, often demonstrating heterogeneous hypointensity on T1 compared to adjacent epidural fat, indicating the degree of vascularity of the tumor. Klisch et al have also noted that signal suppression on T1-weighted fat-saturation sequences may be useful in differentiating angiolipoma from subacute hemorrhage or melanoma. ${ }^{14}$ On T2 images, the lesions usually appear hyperintense. Avid post-gadolinum enhancement is also seen with highly vascular lesions, such as in this case. ${ }^{13}$

Macroscopic pathological samples for SAL exhibit reddishyellow, soft masses with or without a capsule, which may infiltrate into adjacent bone. Histologically, SAL is comprised of mature adipocytes with peripheral, sometimes indented nuclei and blood vessels with variable size and no atypia or mitosis. The ratio of fat to vessel is in the range of $1: 3$ to $2: 3$, with the vasculature often containing fibrin thrombi, which are absent in typical lipomas. ${ }^{15,16}$ When an abundance of smooth muscle fibers are noted on microscopy, the lesion is further sub-classified as angiomyolipoma. ${ }^{6,17}$ The features of the lesion can also mimic capillary angioma, cavernous angioma, or arteriovenous malformations.

The preferred treatment for SAL is complete surgical excision. In cases of dorsally located, non-infiltrating lesions a gross total resection of the tumor and associated capsule is usually achievable. In infiltrating lesions, total removal of nonepidural tumor is often difficult due to the often highly vascular nature of these lesions. In these cases, pre-operative angiography and embolization followed by an anterior approach has been described and may provide an opportunity for greater spinal decompression and tumor removal. Even with subtotal resection, however, the prognosis is excellent with only one reported case of recurrence thus far in the literature. ${ }^{3,4}$ As these lesions are histopathologically benign and slow growing, no adjuvant therapy in the form of chemotherapy or radiation is required and once adequate surgical decompression of neural elements has been achieved, no further treatment is required.

In conclusion, spinal angiolipomas are rare benign neoplasms that cause progressive neurological deficit from compression of neural structures. We present the case of a patient who developed myelopathy secondary to epidural compression of the thoracic cord. In keeping with prior documented cases of this lesion, the patient's symptoms improved significantly after gross-total resection of the offending lesion and no further adjuvant therapy was required. This report reaffirms the importance of understanding the clinical, radiological and pathological characteristics of angiolipomas, such that it can be included in the differential diagnosis of patients presenting with epidural tumours in the spinal canal.

\section{REFERENCES}

1. Von Hanwehr R, Apuzzo MLJ, Ahmadi J, Chandrasoma P. Thoracic spinal angiomyolipoma: case report and literature review. Neurosurgery. 1985;16:406-11.

2. Rabin D, Pelz DM, Ang LC, Lee DH, Duggal N. Infiltrating spinal angiolipoma- a case report and review of the literature. J Spinal Disord Tech. 2004;17:456-61.

3. Hungs M, Pare LS. Spinal angiolipoma: case report and literature review. J Spinal Cord Med. 2008;31:315-18.

4. Yen $\mathrm{H}$, Tsai $\mathrm{S}$, Liu S. Infiltrating spinal angiolipoma. J Clin Neurosci. 2008;15:1170-3.

5. Berenbruch K. Ein Fall von multiplen Angiolipomen kombi- niert mit einem Angiom des Rü ckenmark. Tü bingen, Germany: Medical Faculty Universita"t Tu"bingen; 1890.

6. Liebscher C. Angiolipom des Wirbelcanals mit Compression des Rückenmarkes. Prag Med Wochenschriften. 1901;26:189-91.

7. Howard WR, Helwig EB. Angiolipoma. Arch Dermatol. 1960;82: 924-31.

8. Guzey FK, Bas NS, Ozkan N, Karabulut C, Bas SC, Turgut H. Lumbar extradural infiltrating angiolipoma: a case report and review of 17 previously reported caseswith infiltrating spinal angiolipomas. Spine J. 2007;7:739-44.

9. Gelabert- - Gonzalez M, Garcia-Allut A. Spinal extradural angiolipoma: report of two cases and review of the literature. Eur Spine J. 2009;18:324-35

10. Ehni G, Love JG. Intraspinal lipomas: case reports, literature review, and clinical and pathologic studies. Arch Neurol Psychiat. 1945;53:1.

11. Bucy PC, Ritchey H. Klippel-Feil's syndrome associated with compression of the spinal cord by an extradural hemangiolipoma. J Neurosurg. 1947;4:1.

12. Bender JL, Van Landingham JH, Manno NJ. Epidural lipoma producing spinal cord compression: report of two cases. J Neurosci. 1974;41:100-3.

13. Park JH, Jeon SR, Rhim SC, Roh SW. Lumbar spinal extradural angiolipoma: case report and review of the literature. J Korean Neurosurg Soc. 2008;44:265-7.

14. Klisch J, Spreer J, Bloss HG, Baborie A, Hubbe U. Radiological and histological findings in spinal intramedullary angiolipoma. Neuroradiology. 1999;41(8):584-7.

15. Chung EB, Enzinger FM. Benign lipoblastomatosis: an analysis of 35 cases. Cancer. 2006;32:482-92.

16. Alaggio R, Coffin CM, Weiss SW, et al. Liposarcomas in young patients: a study of 82 cases occurring in patients younger than 22 years of age. Am J Surg Pathol. 2009;33:645-58.

17. Akhaddar A, Albouzidi A, Elmostarchid B, Gazzaz M, Boucetta M. Sudden onset of paraplegia caused by hemorrhagic spinal epidural angiolipoma: a case report. Eur Spine J. 2008;17: S296-8. 